Disclosures Y. Liu: None. W. Brinjikji: None. M. Abbasi: None. D. Dai: None. J. Arturo Larco: None. D. Kallmes: None. L. Savastano: None.

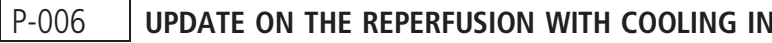 CEREBRAL ACUTE ISCHEMIA II (RECCLAIM II) TRIAL}

${ }^{1} \mathrm{R}$ Gupta*, ${ }^{2} \mathrm{~S}$ Zaidi, ${ }^{2} \mathrm{M}$ Jumaa, ${ }^{3} \mathrm{~N}$ Badjatia, ${ }^{4} \mathrm{~A}$ Yoo. ${ }^{1}$ WMG Neurosurgery, Wellstar Health System, Marietta, GA; ${ }^{2}$ Department of Neurology, University of Toledo Health Sciences Campus, Toledo, $\mathrm{OH}_{;}{ }^{3}$ Department of Neurology, University of Maryland, Baltimore, MD; ${ }^{4}$ Department of Radiology, Texas Stroke Institute, Plano, TX

\subsection{6/neurintsurg-2021-SNIS.42}

Introduction Endovascular therapy for large vessel occlusion has become the standard of care for appropriate patients treated under 24 hours from onset. Despite being superior to standard medical management, roughly $50 \%$ of patients still do not achieve independent neurological functioning. Therapeutic hypothermia (TH) impacts multiple pathways to provide neuroprotection in stroke by lower metabolic demand. Prior trials with $\mathrm{TH}$ may have failed due to inconsistent reperfusion therapies at that time. The RECCLAIM II study was designed to test if the addition of $\mathrm{TH}$ to endovascular reperfusion is feasible and safe. We intend to provide an update on the progress and status of the study as enrollment continues.

Methods Prospective, multi-center, randomized-controlled study with a target enrollment of 120 patients to assess the safety and feasibility to achieve rapid hypothermia to under 34 degrees $\mathrm{C}$ with the ZOLL Intravascular Proteus system prior to achieving reperfusion. Cooling will be maintained for 6 hours with rewarming to 36.5-37 degrees $\mathrm{C}$ over the next 3-6 hours. CT studies will be assessed for hemorrhagic complications 24 hours post-procedure and 90-day blinded outcomes will be obtained on each patient. Up to ten centers will enroll up to 120 patients for the study. Each center will perform 4 Roll-in cases prior to entering the randomization phase. The primary feasibility endpoint is that the majority of patients will achieve a temperature of 34 degrees $\mathrm{C}$ within one hour of arterial puncture without significant delays to reperfusion when comparing the control and treatment arms. The secondary endpoints include rates of hemorrhage and clinical outcomes in both arms.

Results A total of 30 patients have been enrolled to date. Times for each procedure, ability to achieve rapid hypothermia, 90 day blinded Modified Rankin, and core lab adjudicated hemorrhagic complication rates will be evaluated. The DMC will review significant adverse events and mortalities. Workflow time metrics will be assessed for both arms to assess for how quickly $\mathrm{TH}$ can be commenced in conjunction with thrombectomy. These results will serve as the basis for powering a phase III study to assess the efficacy of $\mathrm{TH}$ in conjunction with reperfusion therapy compared to reperfusion therapy alone.

Conclusion There is limited evidence demonstrating the clinical benefit or impact of neuroprotection in conjunction with endovascular reperfusion therapy for acute ischemic stroke. Combining intravascular therapeutic hypothermia with endovascular reperfusion therapy may provide an opportunity to enhance clinical outcomes in patients with large vessel occlusion.
Disclosures R. Gupta: 1; C; Stryker Neurovascular PI ASSIST Registry, Zoll PI RECCLAIM II (No compensation), Cerenovous Steering Committee MEMBRANE study, Medtronic Steering Committee ELEVATE Study, Penumbra CEC MIND Trial, Vesalio PI CLEAR Study, Rapid Medical PI Tiger Study. S. Zaidi: None. M. Jumaa: None. N. Badjatia: None. A. Yoo: 1; C; Medtronic, Cerenovous, Penumbra, Stryker, Genentech. 2; C; Cerenovous, Genentech. 6; C; Insera Therapeutics.

\section{P-007 SAFETY AND EFFICACY OF ENDOVASCULAR THROMBECTOMY FOR DISTAL VESSEL OCCLUSION (DLV): SINGLE CENTER EXPERIENCE}

S Majidi*, S Matsoukas, R De Leacy, T Oxley, H Shoirah, T Shigematsu, C Kellner, J Fifi, J Mocco. Neurosurgery, Mount Sinai Hospital, New York, NY

\subsection{6/neurintsurg-2021-SNIS.43}

Background Endovascular thrombectomy is the gold standard treatment for emergent proximal large vessel occlusion stroke. The safety and efficacy of thrombectomy in distal branch occlusion is not well understood. We aimed to evaluate the technical safety and feasibility of thrombectomy in distal vessel occlusion (DVO).

Methods We performed a retrospective analysis of consecutive acute stroke patients with distal intracranial occlusion (defined as M3, A2 or P2 occlusion) who underwent thrombectomy within 24 hours from the time of last known well. The primary efficacy outcome was successful reperfusion (TICI $\geq 2 \mathrm{~B}$ ). Secondary outcomes included successful recanalization with $\leq 3$ passes. The safety outcome included the rate of subarachnoid hemorrhage (SAH), all intracranial hemorrhages (ICH) and symptomatic $\mathrm{ICH}(\mathrm{sICH})$.

Results Total of 72 patients with acute DVO treated with thrombectomy were identified. The median (IQR) age was 70 (20), 38\% women, and 21\% African-American. The site of occlusion included M3 (54\%), P2 (28\%), and A2 (18\%) segments of middle, posterior and anterior cerebral arteries, respectively. Admission NIHSS score median (IQR) was 12 (11), and $90 \%$ of the patients had baseline mRS $\leq 2$. Thirty six percent of the patients had received intravenous thrombolytic therapy. Successful recanalization was achieved in $90 \%$ of the patients. Forty six percent of the procedures were performed under general anesthesia. Direct aspiration technique was used in $64 \%$ of the patients. The median number of passes was 2, with successful thrombectomy achieved with $\leq 3$ passes in $83 \%$ of the patients. ICH was seen in $16 \%$ of the patients, including $3 \mathrm{SAH}$. However, only one patient $(1.3 \%)$ had sICH. Among 48 patients in whom 90-day outcome data was available, $44 \%$ had favorable clinical outcome $(\mathrm{mRS} \leq 2)$.

Conclusion This single center, consecutive real-world experience demonstrates that thrombectomy in DVO stroke patients is safe and leads to high rate of successful recanalization. Further studies are warranted to evaluate the efficacy of thrombectomy in DVO in comparison to best medical management. Disclosures S. Majidi: None. S. Matsoukas: None. R. De Leacy: None. T. Oxley: None. H. Shoirah: None. T. Shigematsu: None. C. Kellner: None. J. Fifi: None. J. Mocco: None. 